\title{
Variable stars: Which Nyquist frequency?
}

\author{
L. Eyer and P. Bartholdi \\ Observatoire de Genève, CH-1290 Sauverny, Suisse
}

Received April 27; accepted August 24, 1998

\begin{abstract}
In the analysis of variable stars, the problem of sampling is central. This article focusses on the determination of the Nyquist frequency. It is well defined in the case of regular sampling. However, the time series of variable stars observations are generally unevenly sampled. Fourier analysis using the spectral window furnishes some clues about the equivalent Nyquist frequency in the irregular case. Often it is pushed very high, and thus very short periods can be detected. A specific example is shown, drawn from MACHO databases.
\end{abstract}

Key words: stars: variable — methods: analytical methods: data analysis

\section{Introduction}

When variable stars observations are analyzed, two major problems arise. First, there are constraints on the sampling times. They can't always be chosen, because of weather conditions or other observing restrictions. Secondly, the stars, even if they are periodic, can have an extremely wide range of periods and behaviors. Therefore, astronomers would like to know up to which higher limit it makes sense to search for frequency.

In the literature, there are contradictions regarding the Nyquist frequency for irregularly sampled data. Some authors (Press et al. 1992; Horne et al. 1986) identify it with $1 / 2 \overline{\delta t}$ where $\overline{\delta t}$ is the "average" sampling rate. The Nyquist frequency is also often identified with $\nu=$ $1 / 2 s$, where $s$ is the smallest time interval in the sample (Scargle 1982; Roberts et al. 1987). Nevertheless, Press et al. (1992) and Roberts et al. (1987) remarked that frequencies can be detected above their quoted values.

For evenly sampled data, the Nyquist frequency is defined as $1 / 2 \delta t$, where $\delta t$ is the time sampling step. We will show how this quantity can be extended to the irregular situation. In most cases, the Nyquist frequency is

Send offprint requests to: L. Eyer much larger than usually thought. The present work is particularly relevant to large databases that are becoming available (MACHO, OGLE, EROS, HIPPARCOS, etc.).

\section{Nyquist frequency for irregular sampling}

Let $f(t)$ be a signal function of time $t$. We suppose that the power spectrum of the function $f$ is not variable in time (it should be noted that this hypothesis is stronger than stationarity). This is the case for periodic functions. Otherwise, the Nyquist frequency is only defined very locally and loses its sense for a given irregular sampling. Moreover, a spectral analysis of an unstable signal which is undersampled may lead to wrong conclusions.

If $f$ is sampled at time $t_{i}$ we are left with $\left(f_{i}, t_{i}\right) i=$ $1, \ldots, N$, where $N$ is the total number of measurements. In the frequency domain, Deeming $(1975,1976)$ showed how the irregular discretization affects the power spectrum $F_{N}(\nu)$ : the spectrum results from the convolution between the real spectrum $F(\nu)$ and the spectral window $G_{N}(\nu)$. $G_{N}(\nu)$ is the Fourier transform of $g(t)=\sum \delta\left(t-t_{i}\right)$. That is $F_{N}(\nu)=F(\nu) \otimes G_{N}(\nu)$.

We can state the result as follows. Let $^{1} p$ be the largest value such that $\forall t_{i}, t_{i}=t_{1}+n_{i} p$, where $n_{i} \in \mathbb{N}$. $p$ is a kind of greatest common divisor (gcd) for all $\left(t_{i}-t_{1}\right)$. The Nyquist frequency then is:

$\nu_{\mathrm{Ny}}=\frac{1}{2 p} \geq \frac{1}{2 s}$.

In most practical cases $p \ll s$, therefore, even with a strong but random under-sampling, very high frequencies can be detected.

\section{Proof}

The key function is the spectral window:

$G_{N}(\nu)=\frac{\left|\sum_{k=1}^{N} \exp \left(i 2 \pi \nu t_{k}\right)\right|^{2}}{N^{2}}$.

\footnotetext{
1 Note that if the $t_{i}$ are irrational, $p$ may not exist and should then be taken as zero.
} 


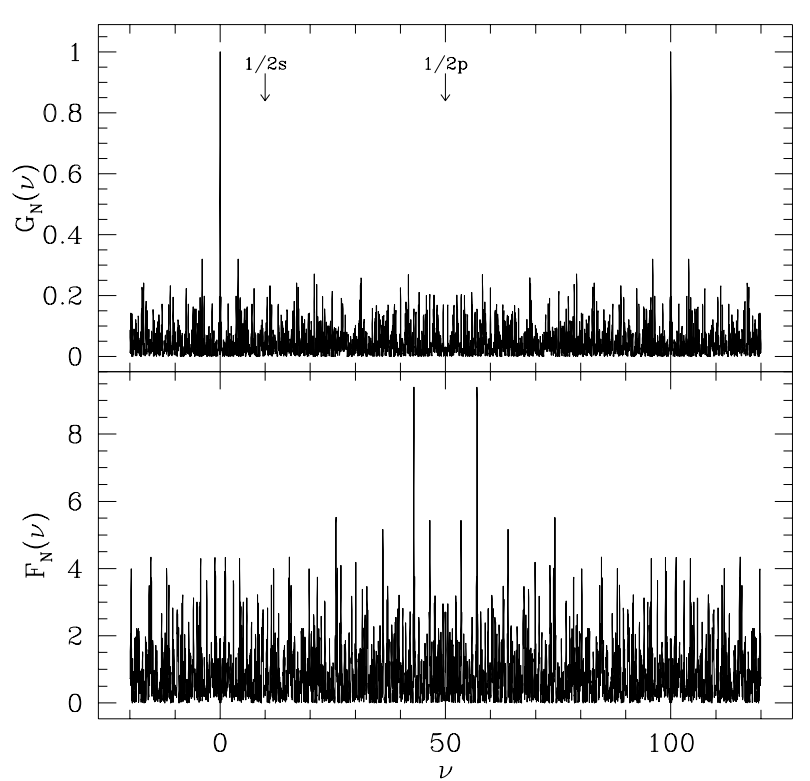

Fig. 1. Spectral window $G_{N}$ and power spectrum $F_{N}$ for the simulation described in the text. Both functions are clearly symmetrical around $\nu_{\mathrm{Ny}}=1 / 2 p=50$. No peak appears for $G_{N}(\nu)$ at $\nu=1 / s$

We have $G_{N}(\nu) \leq 1$ and $G_{N}(\nu)=1$ for $\nu=m / p, m \in \mathbb{N}$. Replacing $t_{\mathrm{k}}$ with $n_{\mathrm{k}} p+t_{1}$, we notice that this function is periodic with period $1 / p$ (each individual term in the sum has this same periodicity). Moreover as $g(t)$ is real, the function $G_{N}(\nu)$ is symmetric around the origin. These properties of periodicity and symmetry imply that $G_{N}(\nu)$ is also symmetric around $(2 k+1) / 2 p$. For $k=0$, we have $1 / 2 p$, which is just the Nyquist frequency. For the power spectrum, the proof is exactly the same. We can think of the set $t_{i}$ as a regular sampling with step $p$, where some (most) data have been dropped. The more random the data is, the smaller $p$ will be and so the higher $\nu_{\mathrm{Ny}}$. As in the regular case, the continuous signal $f(t)$ should not contain any component above $\nu_{\mathrm{Ny}}$. Otherwise, it will be mirrored into $2 \nu_{\mathrm{Ny}}-\nu$, and, without further assumptions, it will not be distinguishable from the mirror image. Of course, no frequency component filtered during the measurement process can be recovered by the random sampling. In particular, if $\Delta t_{\exp }$ is the exposure time of every measures, then in the frequency domain, the signal is filtered by $\sin ^{2}\left(\pi \Delta t_{\exp } \nu\right) /\left(\pi \Delta t_{\exp } \nu\right)^{2}$ and not much information will be recovered above $\nu_{\max }=1 /\left(\kappa \Delta t_{\exp }\right), \kappa=$ 2 or 3 .

\section{Simulation}

The following numerical experiment illustrates the theory presented above. We chose the times as

$t_{\mathrm{k}}=\frac{\lfloor 1000 \cdot \mathrm{rnd}\rfloor}{100}, k=1, \ldots, 20$

where rnd are random numbers uniformly distributed on $\left[0,1\left[\right.\right.$. Then, we built up a signal as follows $f\left(t_{i}\right)=$ $3 \sin \left(2 \pi \nu_{0} t_{i}\right)$, with $\nu_{0}=43($ period $=.0233 \ldots)$.

\begin{tabular}{|lr||lr|}
\hline Time & Signal & Time & Signal \\
\hline 0.00 & -2.69 & 5.59 & 0.88 \\
0.52 & 0.70 & 6.12 & -2.55 \\
1.07 & -2.77 & 7.27 & 2.91 \\
1.33 & -2.21 & 7.84 & -2.86 \\
1.77 & -2.91 & 8.13 & 2.98 \\
2.00 & -2.69 & 8.32 & 1.14 \\
2.77 & -2.91 & 8.49 & -2.99 \\
3.31 & 0.14 & 8.54 & -1.80 \\
4.30 & -1.40 & 9.00 & -2.69 \\
4.49 & -2.99 & 9.29 & 2.39 \\
\hline
\end{tabular}

The smallest time interval is $s=0.05$, so $\nu_{0}$ is well above $1 / 2 s$, but $p$ is 0.01 . As expected, we observe that the Nyquist frequency is at 50, and that there is no strong aliasing peak before $\nu=100$ (Fig. 1).

If we had rounded the times $t_{\mathrm{k}}$ to 0.001 in the above example and had $p$ at 0.001 , we would have found $\nu_{\mathrm{Ny}}$ at 500.

\section{Determination of $p$}

Theoretically, $p$ could be found using an extension to more than 2 numbers of the Euclid gcd algorithm. But in practice, the $t_{\mathrm{k}}$ are not known to infinite precision, and the Euclid algorithm is numerically unstable for non integer numbers. A practical solution could then be to stop the algorithm when the smallest number is smaller than some $\epsilon$. Otherwise, the spectral window is probably the most efficient way to find $p$ in this fuzzy environment. $p$ is the inverse of the smallest $\nu$ for which $G_{N}(\nu)$ is greater than some value just below one.

\section{Application}

Actually, most data are obtained at approximately equal time intervals. The fact that there is some randomness may raise substantially the Nyquist frequency. As a conspicuous example, we can cite Minniti et al. (1998) who are able to find periods for $\delta$ Scuti stars with MACHO observations of bulge fields. Indeed, the data are separated by about one day and the periods found are of few hours. We give an example for the $\delta$ Scuti star 162.25348.3066. The histogram of the time intervals is given in Fig. 2, its Fourier transform is shown in Fig. 3, and finally the folded curve is presented in Fig. 4, it leaves no doubts that the period is the right one. The detection limit is dominated by the exposure time $(150 \mathrm{~s})$, that is about $\nu_{\max }=$ 192 days $^{-1}$ if $\kappa=3\left(P_{\min }=7.5 \mathrm{~min}\right)$.

With the HIPPARCOS mission the smallest time interval is 20 minutes. A search for shorter periods was undertaken (Eyer 1998). Unfortunately no results came out. We can invoke that often the spectral window is nearly symmetric around $1 / 40 \mathrm{~min}^{-1}$ (this is due to the fact that observations are often found in sequences, inducing strong 


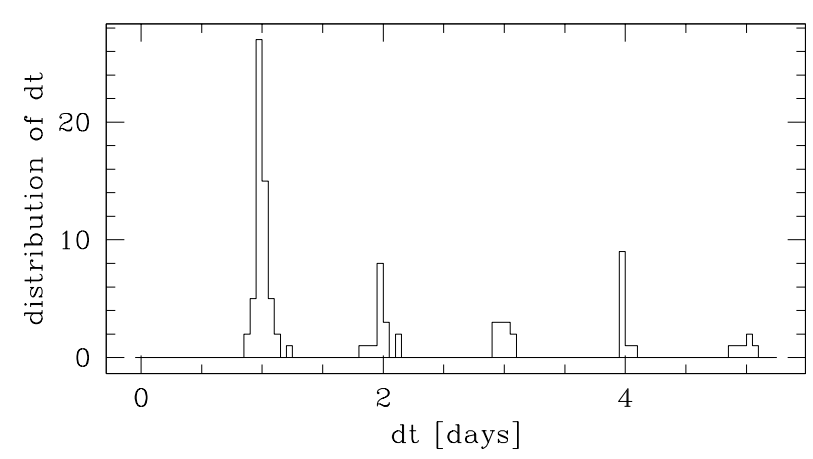

Fig. 2. Histogram of the time intervals. The smallest is around 0.8 days. In total, 122 measurements were available

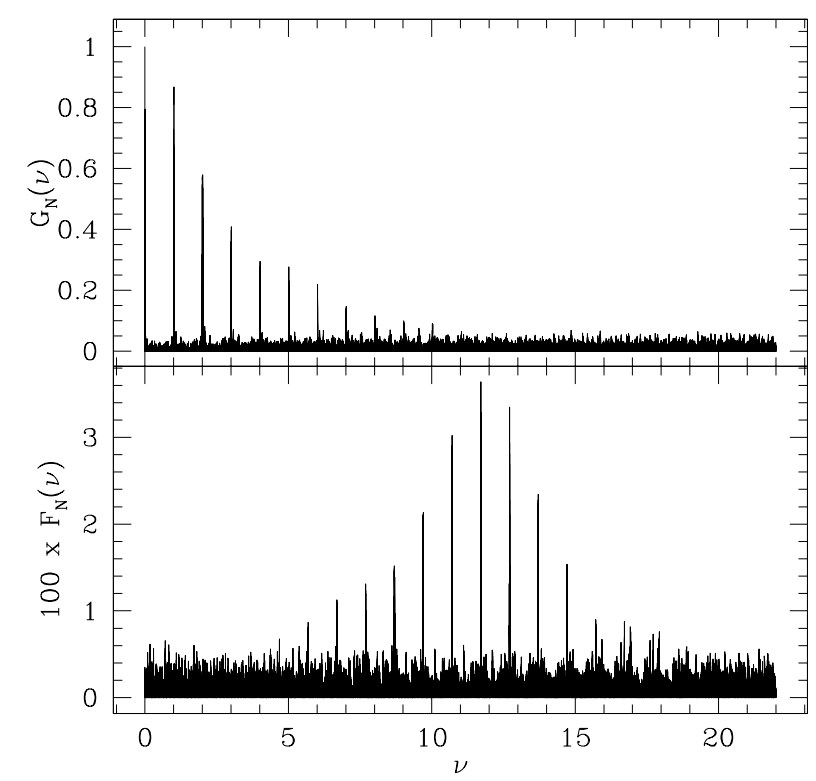

Fig. 3. Spectral window $G_{N}$ and power spectrum $F_{N}$ of the $\delta$ Scuti star 162.25348.3066 from MACHO. Although there are rather strong aliasing peaks, the frequency of $1 / 0.0854259$ days $^{-1}$ is unambiguous

aliasing peaks), or that short periods are rarely stable over three years (either period change or phase shift can occur).

\section{Reducing the amplitude of peaks in $G_{N}(\nu)$}

The spectral window also helps to decide when new measurements should be made to reduce the annoying spikes in the spectral window. We make use of the simulated data in Sect. 4 as an example. A practical procedure could be to determine the polar coordinates $\rho, \theta$ (point (1) in Fig. 5, where we used as a concrete example the simulated data of Sect. 4) of the complex number $(1 / N) \sum_{k=1}^{N} \exp \left(i 2 \pi \nu_{\mathrm{h}} t_{\mathrm{k}}\right)$ for the annoying frequency $\nu_{\mathrm{h}}$. Point (1) is the center of gravity of the observing points. The value of $G_{N}\left(\nu_{\mathrm{h}}\right)$ is the distance of point (1) to the origin. Then, the new measurement should be taken at

$t_{N+1}=\left(k+\frac{\pi+\theta}{2 \pi}\right) \frac{1}{\nu_{\mathrm{h}}}$

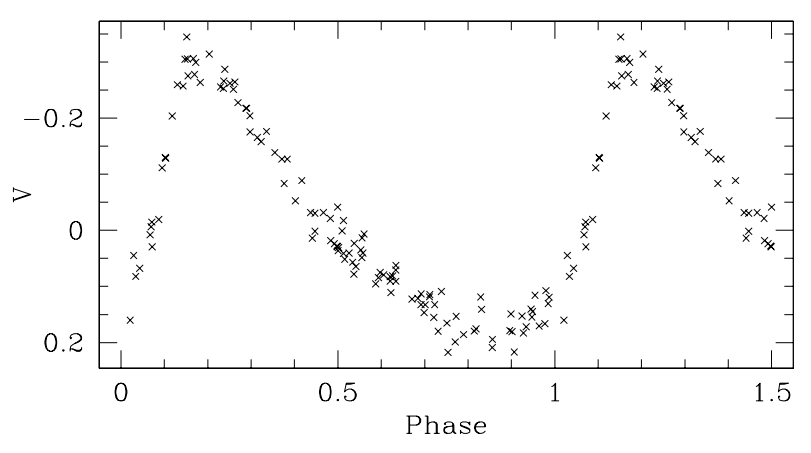

Fig. 4. The folded curve of the $\delta$ Scuti star 162.25348.3066 from MACHO (period $=0.0854259$ days)

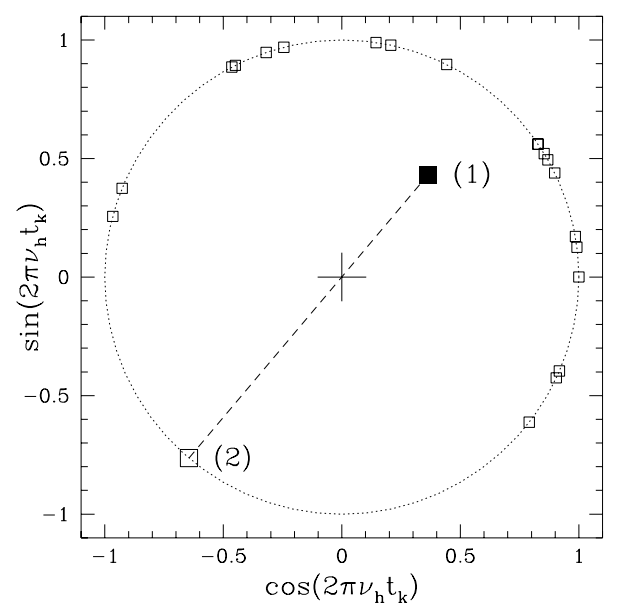

Fig. 5. The points $\exp \left(i 2 \pi \nu_{\mathrm{h}} t_{\mathrm{k}}\right)$ are displayed in the complex plane (small open squares) for the frequency $\nu_{\mathrm{h}}=3.99$ corresponding to the highest peak of $G_{N}(\nu)(1<\nu<50$, in Fig. 1). The black square (1) represents the center of gravity of these points, while the open square (2) opposed to (1) on the circle, is the best position for $t_{N+1}$ (modulo $2 \pi \nu_{\mathrm{h}}$ ) to reduce the amplitude of $G_{N}$ at $\nu_{\mathrm{h}}$

for some integer value $k$ compatible with the other constraints. This produces the largest reduction of the peak.

Acknowledgements. We would like to thank S. Paltani, G. Burki, F. Kienzle, C. Fluetsch, D. Kurtz and the reviewer A. Milsztajn for their interesting discussions and comments. Furthermore, we thank very warmly D. Minniti for his helpful and efficient collaboration.

\section{References}

Deeming T.J., 1975, Astrophys. Space Sci. 36, 137

Deeming T.J., 1976, Astrophys. Space Sci. 42, 257

Eyer L., 1998, PhD Thesis, Geneva University, Switzerland

Horne J.H., Baliunas S.L., 1986, ApJ 302, 757

Minniti D., Alcock C., Alves D.R., et al., 1998, IAU Symp. 189, p. 293

Press W.H., Teukolsky S.A., Vetterling W.T., Flannery B.P., 1992, Numerical Recipes in Fortran. Cambridge University Press

Roberts D.H., Lehár J., Dreher J.W., 1987, AJ 90, 968

Scargle J.D., 1982, ApJ 263, 835 